\title{
Fuzzy Neural Technique Based Dynamic Voltage Restorer for Power Quality Enhancement Under Different Voltage Variations
}

\author{
Abdul-Jabbar Fathel Ali ${ }^{1} \quad$ Wael Hussein Zayer ${ }^{2} \quad$ Samhar Saeed Shukir ${ }^{3}$ \\ ${ }^{1}$ Electrical Engineering Department, Wasit University, Kut-Iraq, AJF_572@ Yahoo.com \\ ${ }^{2}$ Electronic Engineering Department, Southern Technical University, Amaraa-Iraq, Wael_zayer@Yahoo.com \\ ${ }^{3}$ Electrical Engineering Department, Middle Technical University, Kut-Iraq, samharalwandi@gmail.com
}

\begin{abstract}
Recently a large interest has been focused on power quality field due to: disturbances caused by non-linear loads, Increase in the number of electronic devices and renewable energy sources. Power quality measures the fitness of the electric power transmitted from generation to the industrial, domestic and commercial consumers. In a power system voltage distortion introduced by harmonics and voltage sags are the most severe affecting power quality, because of both consumers and utilities are affected by these disturbances. Different methods to enhance the power quality but the custom power device is the most effective and efficient method. One of which is the use of Dynamic Voltage Restorer (DVR). The performance of the DVR based on Fuzzy Neural controller to restore the load voltage to its nominal value under different fault conditions and power disturbances is presented in this paper. Simulation results are carried out using MATLAB/SIMULINK program. The faults and disturbances are initiated at $0.8 \mathrm{~s}$ and kept till $0.95 \mathrm{~s}$.
\end{abstract}

Keywords-Dynamic voltage restorer; Voltage variations compensation; Artificial intelligent; Fuzzy Neural controller; Power quality improvement

\section{INTRODUCTION}

Electrical power is the main element that required in any function in the commercial and industrial sectors therefore it should be available at all times. Power quality is a set of parameters that define the characteristics of the power supply as delivered to the consumers in normal operating conditions such as: continuity of supply, frequency, magnitude of voltage, waveform and symmetry. Recently, power quality is not only a technical problem but also a problem that leads to financial issues. Many surveys have been shown that poor power quality causes large economic losses to industrial sectors. Moreover, large amount of power is wasted due to poor power quality in the services, also putting life at risk where safety is priority. Voltage sag, harmonics, voltage swell and voltage interruption are the most common power quality problems in the industrial, service and transparent sectors. Sag is a sudden decrease in the r.m.s voltage and make it between $10 \%$ and $90 \%$ from its nominal value, and lasting from 0.5 cycle to several seconds. Sag can be symmetrical or unsymmetrical, a three-phase fault produces symmetrical sags, while double line to ground fault and single line to ground fault cause unsymmetrical sag. Harmonics is the presence of spectral components whose frequencies are multiplies the fundamental frequency. The harmonics occur due to non-linear loads such as electronic devices and induction motors. According to IEEE standards for the system of less than $69 \mathrm{kV}$ the harmonic distortion limits are 3\% for individual voltage and 5\% for total harmonic distortion [1]. Harmonics cause overheating, overloading and additional losses to the transformers, motors and other power system equipment. A Swell is a sudden increase in the r.m.s voltage and make it between $110 \%$ and $190 \%$ from its nominal value and lasting from 0.5 cycle to several seconds. The main reason for the swell is turn on large capacitive loads or shutdown large inductive loads. The voltage interruption is decreasing the voltage to less than $10 \%$ from its nominal value for a period not exceeding $1 \mathrm{~min}$. To solution the power quality problems there are different methods such as: APF (active power filter), Dynamic voltage restorer (DVR), SVC (static var compensator), DSTATCOM (distribution static synchronous compensator), BESS (battery energy storage system), TSC (thyristor switched 
capacitor), UPS (uninterrupted power system) [2]. DVR is one of the most efficient and reliable solutions due to the lower cost, smaller size and dynamic response, and DVR is effective in restoring the load voltage to the nominal value and make it regular under all cases of faults and non-linear conditions. The first DVR in the world was installed in the USA in the year 1996. It was engineered by Electrical Power Research Institute. This DVR was installed at the medium voltage level of $12 \mathrm{kV}$ and rated for 2MVA [3]. The DVR is a quick and a flexible solution to electrical power problems it can return the load voltage in a few milliseconds and prevent any power disruption to loads. For improving the performance of the (DVR) several studies have been conducted, these studies are shown that outer voltage loop and inner current loop was proposed in control system of the DVR. And the studies are shown that an Artificial Intelligent controller for the DVR is effective and powerful in eliminating the power quality problems because that the inverter in DVR is a non-linear and needs a non-linear controller. The capability of the DVR based on adaptive controller such as fuzzy neural optimized fuzzy logic controller is more efficient and effective in mitigating the power quality problems. The proposed DVR injects the appropriate voltage component to treat and keep the load voltage constant and balance under different power disturbances and fault conditions without any difficulties.

\section{THE LOCATION OF THE DVR}

The connection of the DVR to the distribution system is either at medium voltage $11 \mathrm{Kv}$ as shown in Fig. 1 or at low voltage $0.4 \mathrm{Kv}$ as shown in Fig.2.

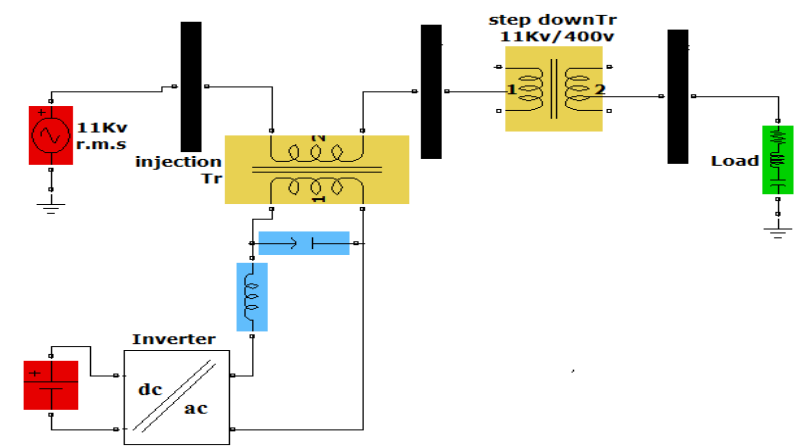

Fig.1. The connection of the DVR at the medium voltage.

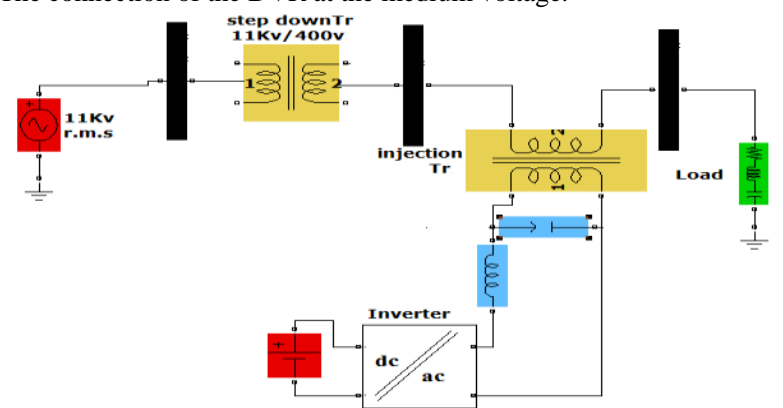

Fig.2. The connection of the DVR at the low voltage.

\section{THE MATHEMATICAL FORMULA OF THE DVR}

DVR is connected in series between the load and the source as shown in Fig.3. 


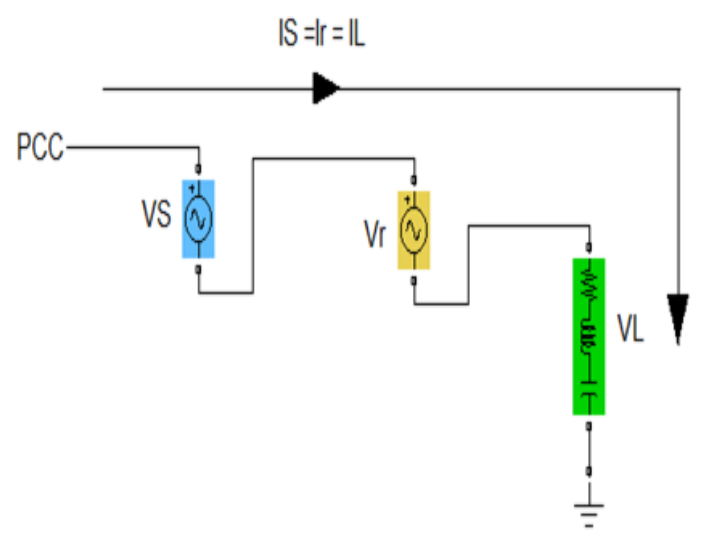

Fig.3. The electrical power circuit with the DVR.

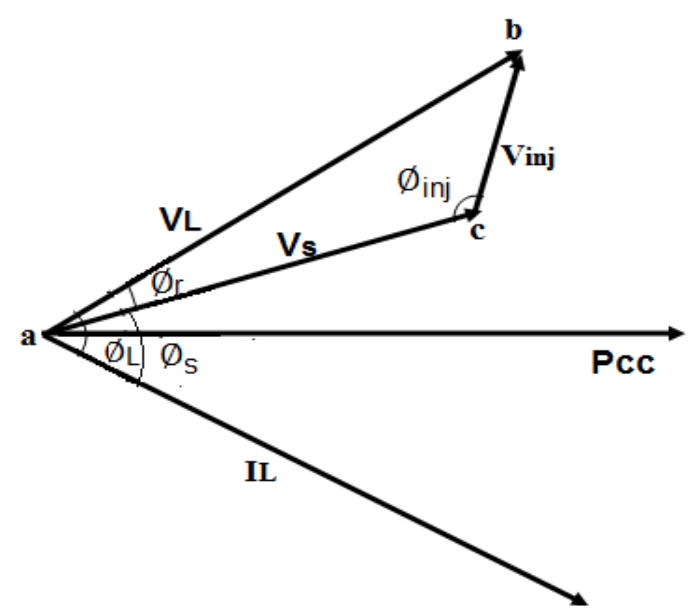

Fig.4. The phasor diagram of the system with DVR.

The power injected by the DVR during the compensation is as follows:

$$
\begin{aligned}
& \mathrm{P}_{\text {DVR }}=\mathrm{P}_{\text {supply }}-\mathrm{P}_{\text {Load }} \\
& \mathrm{Q}_{\text {DVR }}=\mathrm{Q}_{\text {supply }}-\mathrm{Q}_{\text {Load }}
\end{aligned}
$$

From Fig.3 the compensation voltage $\left(\mathrm{V}_{\text {inj }}\right)$ and $\left(\emptyset_{\text {inj }}\right)$ of the DVR can be calculated as follows:

$$
\mathrm{P}_{\mathrm{L}}=\mathrm{V}_{\mathrm{L}} * \mathrm{I}_{\mathrm{L}} * \operatorname{Cos} \emptyset \mathrm{L}
$$

From the triangle abc in Fig.4

$$
\begin{gathered}
\emptyset \mathrm{r}=\emptyset \mathrm{L}-\emptyset \mathrm{s} \\
\mathrm{P}_{\mathrm{r}=} \mathrm{V}_{\mathrm{L}} * \mathrm{I}_{\mathrm{L}} * \operatorname{Cos} \emptyset L-\mathrm{V}_{\mathrm{s}} * \mathrm{I}_{\mathrm{L}} * \operatorname{Cos} \emptyset \mathrm{s}
\end{gathered}
$$

$$
\begin{gathered}
\mathrm{V}_{\text {inj }}=\sqrt{V_{s}^{2}+V^{2}{ }_{L}-2 V_{S} V_{L} \cos \left(\emptyset_{L}-\emptyset_{S}\right)} \\
\emptyset_{\text {inj }}=\operatorname{Sin}^{-1}\left(\frac{\mathrm{VL}}{\operatorname{Vinj}} \operatorname{Sin} \emptyset_{\mathrm{r}}\right)
\end{gathered}
$$

\section{THE CONTROL SYSTEM OF THE DVR}

Control strategy of the DVR plays an important role in its performance [4]. The incorrect injection or the delay in the process would be dangerous to sensitive loads. The major functions of the DVR controller are, detection of voltage disturbances events in the system, calculation of the compensating voltage and generation the reference signal for the PWM to trigger the voltage source inverter [5]. The DVR controller in this paper consists of several functional blocks; i) Fig.5 shows the Phase Locked Loop (PLL) which is synchronized to the fundamental of the transformer primary voltages. 


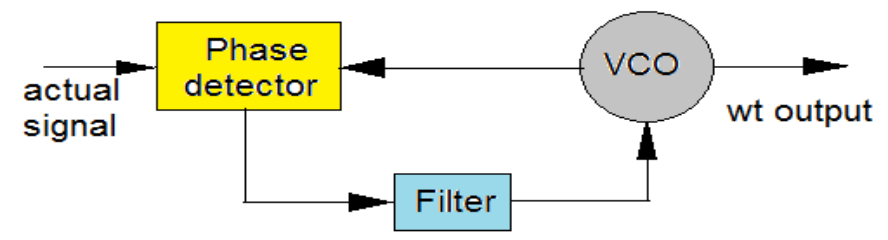

Fig.5. The Phase Locked Loop.

ii) Park transformation is transforming the three $\mathrm{AC}$ quantities $(\mathrm{Va}, \mathrm{Vb}, \mathrm{Vc})$ to two $\mathrm{DC}$ quantities $(\mathrm{Vd}, \mathrm{Vq})$ to simplify the calculations, control, and analysis [6]. iii)Two measurement systems, Vmeas and Imeas blockes.

$$
\begin{aligned}
\mathrm{V}_{\text {meas }} & =\sqrt{\mathrm{V}^{2}{ }_{\mathrm{d}}+\mathrm{V}^{2} \mathrm{q}} \\
\mathrm{I}_{\text {meas }} & =\sqrt{\mathrm{I}^{2}{ }_{\mathrm{d}}+\mathrm{I}^{2} \mathrm{q}}
\end{aligned}
$$

iv) inner current regulation loop consists of one controller; the controller output is the Vq voltage. The $\mathrm{Vq}$ and $\mathrm{Vd}$ voltages are converted into phase voltages $\mathrm{Va}, \mathrm{Vb}, \mathrm{Vc}$ which are used to synthesize the PWM voltages. The Iq reference comes from the outer voltage loop. v) An outer voltage regulation loop consists of one controller to regulate the voltage. Conventional controllers (PI controller, PID controller) tuning is more difficult in non-linear system like DVR, it may lead to many problems such as, complex computation of the DVR control and make the control design complex and uncertainty in these system, therefore it is preferred to use various intelligent techniques available [7].

\section{FUZZY NEURAL OPTIMIZED FUZZY LOGIC CONTROLLER BASED DYNAMIC VOLTAGE RESTORER}

Fuzzy Neural is a combined between a fuzzy qualitative approach and adaptive learning abilities of the neural network, where this system can be trained without a significant amount of expert knowledge that usually required for the standard FL. Jang in 1993 introduced Adaptive Neuro Fuzzy Inference System and mentioned that the (ANFIS) architecture can be utilized to model non-linear functions in control systems and predict a chaotic time series [9]. ANFIS uses the Sugeno-type fuzzy inference system. ANFIS requires for training a set of input and output data, it can choose the parameters of the fuzzy inference system adaptively from the training data. It produces a set of membership functions to map the input data to output. The implementation of the ANFIS uses for tuning input and output a back-propagation method and it uses for learning a hybrid algorithm [10]. In this thesis the advantages of the ANFIS will be defined in modeling and control of a non-linear system.

\section{ANFIS OPTIMIZED FUZZY LOGIC CONTROLLER}

This sugeno fuzzy model has two inputs:( error(e) and change of error $(\Delta e)$ ) and one output which is tuned using the back-propagation method [11] as shown in Fig.6.

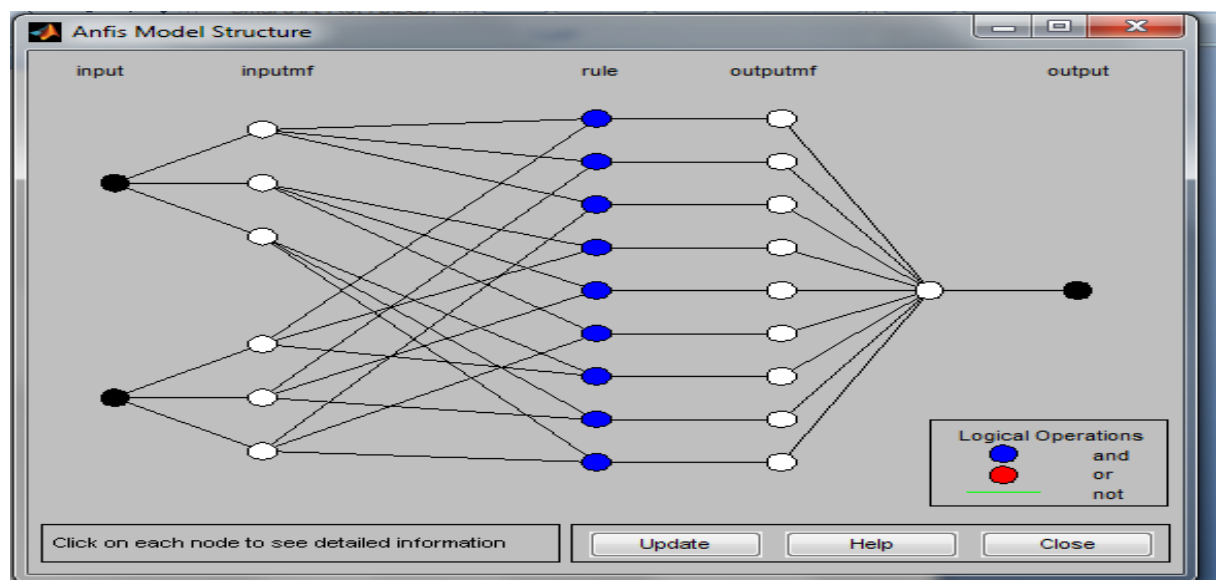

Fig.6. The structure of the ANFIS.

It clears from the figure above that the ANFIS has five layers. The basic principle of these layers is as following [8] 
Layer 1: It is called fuzzification layer and it has six nodes each node is adaptive to an output. If the membership functions in this layer is a triangular function, then the output from each node in this layer is obtained from the equations of the triangular function:

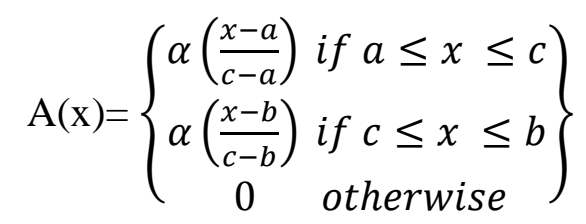

Where the $\mathrm{a}, \mathrm{b}, \mathrm{c}$ is the parameter of the triangular function and $\alpha$ is the cut set. The parameters a, b, $\mathrm{c}$ in this layer is known as premise parameter. This layer calculates the grade of membership for each input $\left(\mu_{\mathrm{Ai}}(\mathrm{x})\right)$. Layer 2: It determines the firing strength for each rule. It has nine nodes (equal to the number of rules). This layer is multiplying the incoming signals from the previous layer. The results are forward to the next layer. output from second layer for each node is:

$$
\mathrm{O}^{2} \mathrm{i}=\mathrm{Wi}=\mu_{\mathrm{Ai}}(\mathrm{x}) * \mu_{\mathrm{Bi}}(\mathrm{y})
$$

Layer 3: It calculates the normalized firing strength for each rule and the output from each node in this layer is equal to the ratio between the firing strength of this node and the summation of all nodes's firing strength Output from each node in third layer is:

$$
\mathrm{O}^{3} \mathrm{i}=\mathrm{Wni}=\frac{W i}{\sum_{j=1}^{j=9} W j}
$$

Layer 4: It determines the output for each rule. Each node in this layer is an adaptive to an output. The output from each node in this layer is obtained by multiplying between the incoming signal from third layer and the set of parameters. Output from each node in the fourth layer is:

$$
\mathrm{O} 4 \mathrm{i}=\mathrm{Wni} * \mathrm{fi}=\mathrm{Wni} *\left(\mathrm{p}_{\mathrm{i}} \mathrm{x}+\mathrm{s}_{\mathrm{i}} \mathrm{y}+\mathrm{t}_{\mathrm{i}}\right)
$$

where ( $\mathrm{p}, \mathrm{s}$ and $\mathrm{t}$ ) are the parameters for each node and it is called the consequent parameter.

Layer 5: This layer determines the output $\mathrm{o} / \mathrm{p}$ by summing the all incoming signals from the fourth layer.

$$
\mathrm{O} / \mathrm{P}=\sum_{i=1}^{i=9} \mathrm{Wni} * \mathrm{fi}
$$

\section{FlOW CHART OF THE ANFIS ALGORITHM}

Fig.7 demonstrates the flow chart of the Adaptive Neuro Fuzzy Inference System.[12]. 


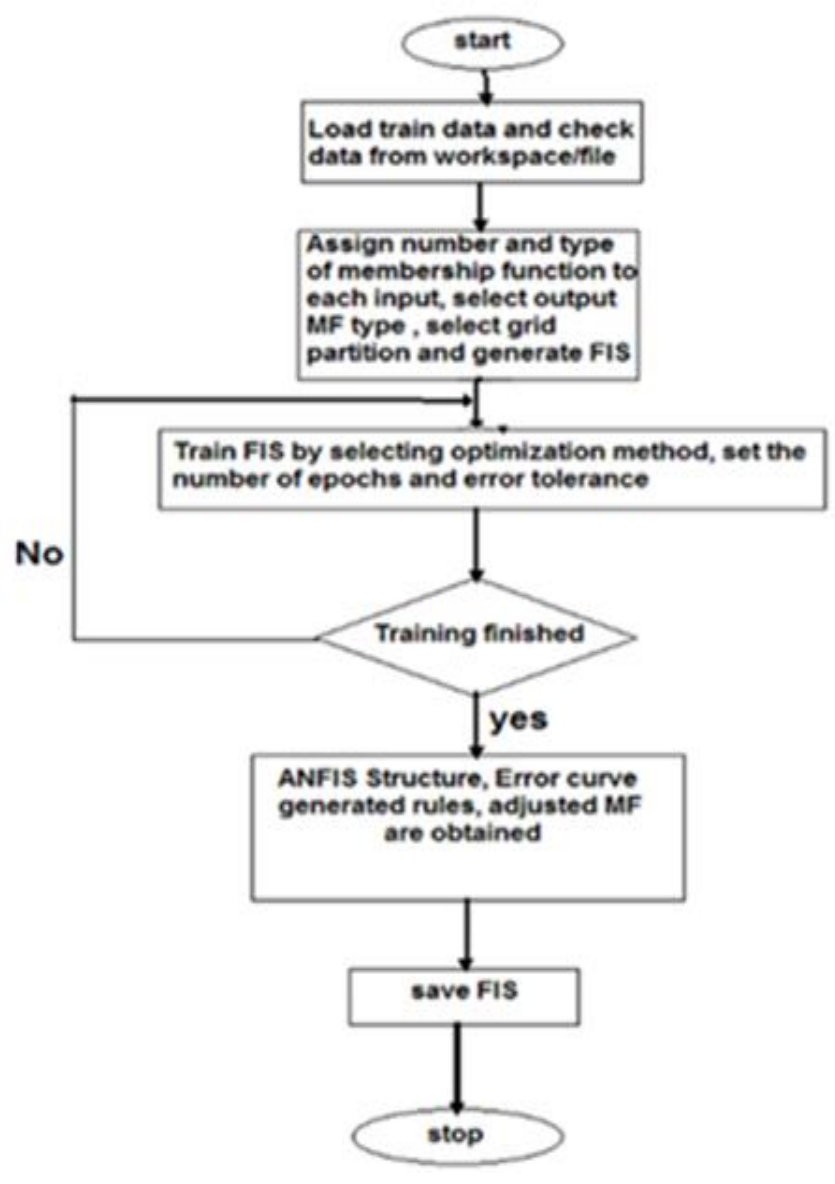

Fig.7. The flow chart of the ANFIS.

\section{MODELING AND SIMULATION}

The modeling and simulation are achieved under different fault conditions. Faults are simulated at $11 \mathrm{kV}$ side in the first feeder as shown in Fig.8 for a periode of $0.15 \mathrm{~s}$ from $0.8 \mathrm{~s}$ till $0.95 \mathrm{~s}$ to show the ability of the DVR in protecting the loads in adjacent feeders from symmrtrical and unsymmetrical sag. Single line to ground fault in the first feeder has no effect on the neighboring feeders in this system, double line to ground fault lead to occure unsymmetrical sag in the adjacent feeders while three phase fault causes to happen a symmetrical sag in the neighboring feeders. The control system of the DVR is shown in Fig.9, it consists of two controllers one for voltage regulation and the other for regulating the current to improve the performance of the Dynamic Voltage Restorer in compensating the required voltage to restore the load voltage to its prefault value. 


\section{V/JIS Wasit Journal}

VOLUME: ( 7 ), NO.:( 3 ) 름ㅁㅁ

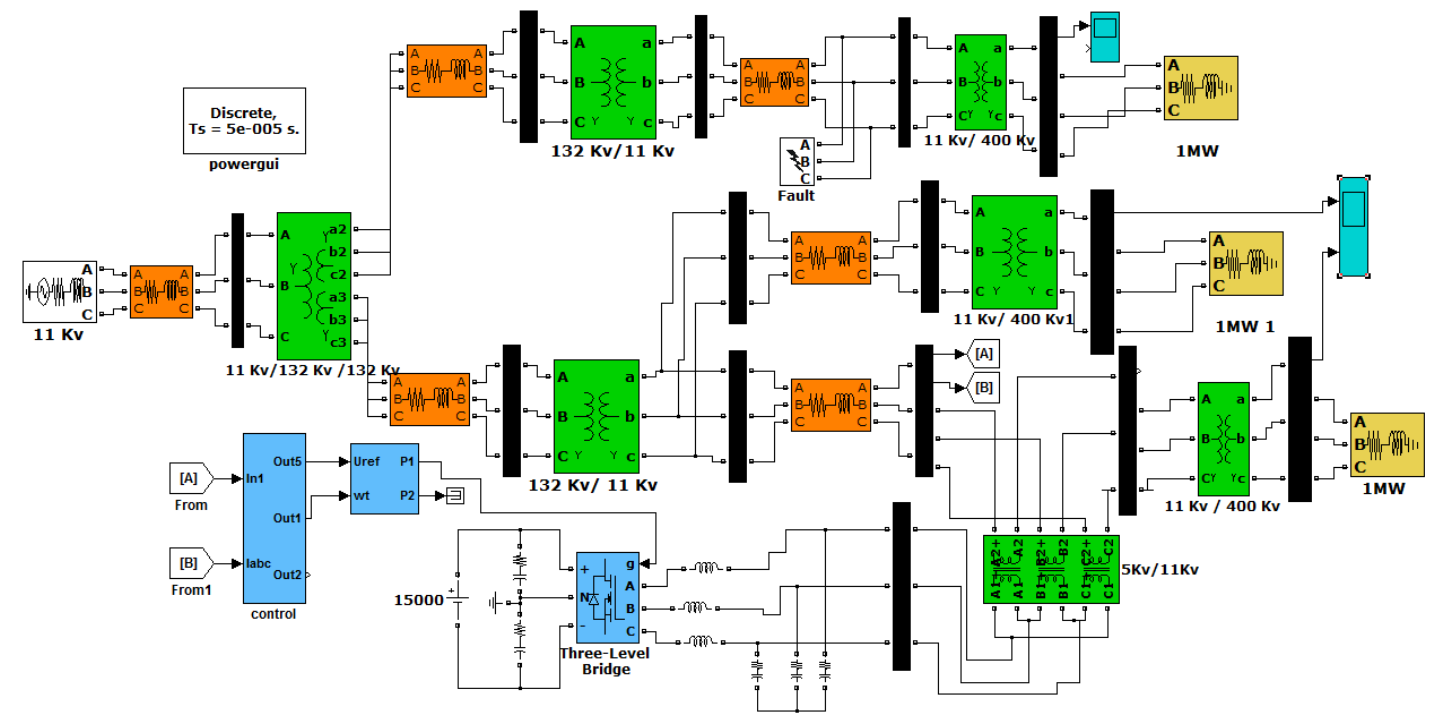

Fig.8. Modeling and simulation the electrical power system with DVR.

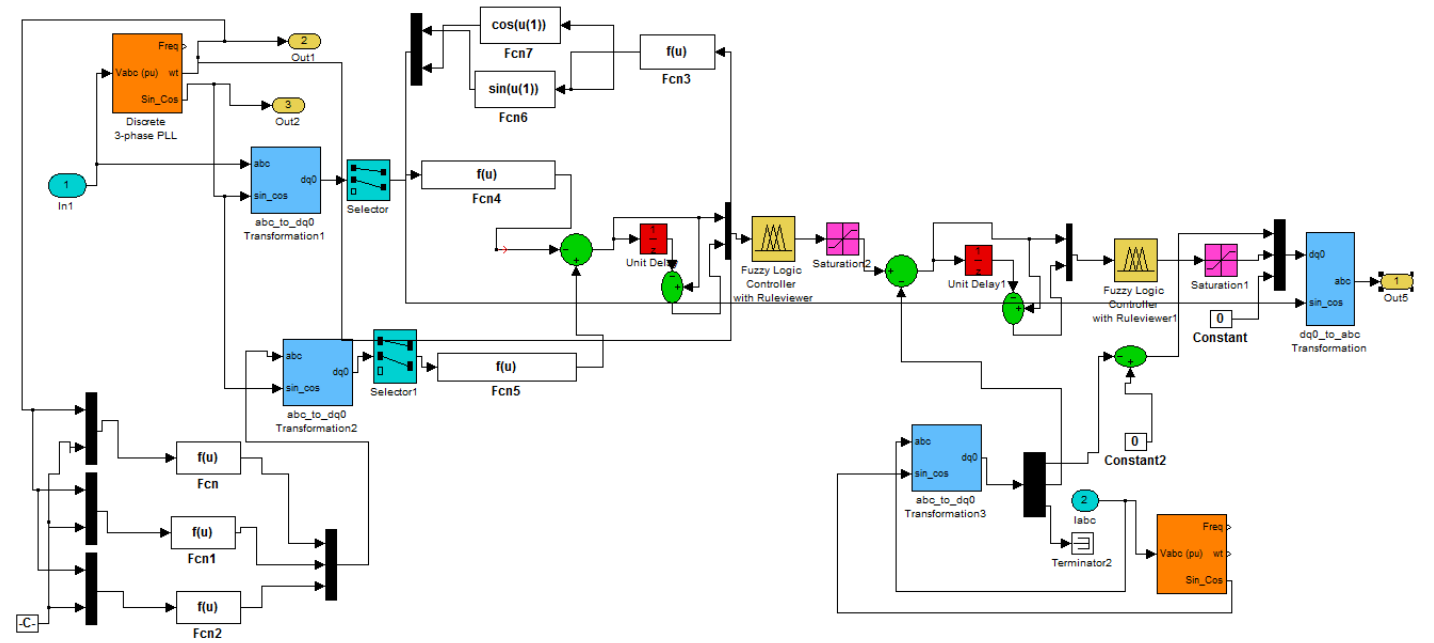

Fig.9. Modeling and simulation the control system of the DVR based on Fuzzy Neural controller.

i)Single line to ground fault

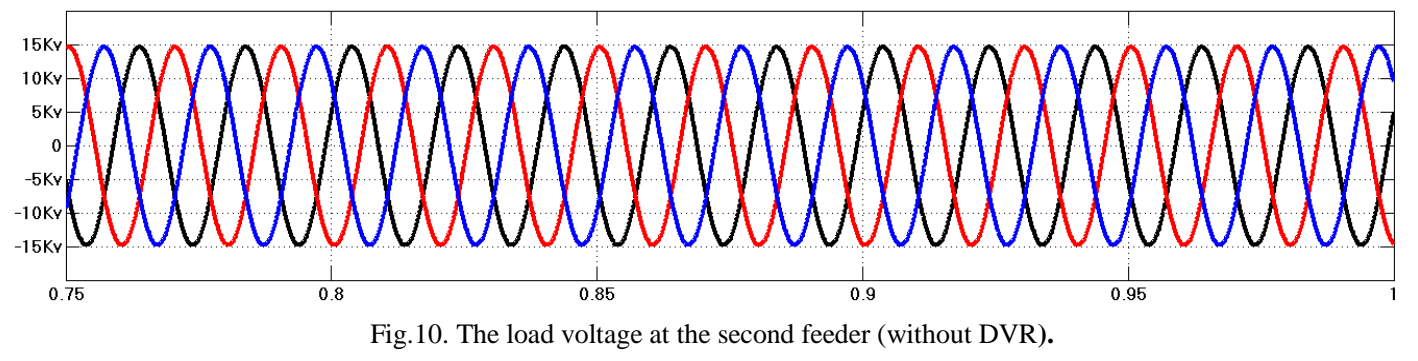




\section{N/JIS Wasit Journal}

VOLUME: ( 7 ), NO.:( 3 )

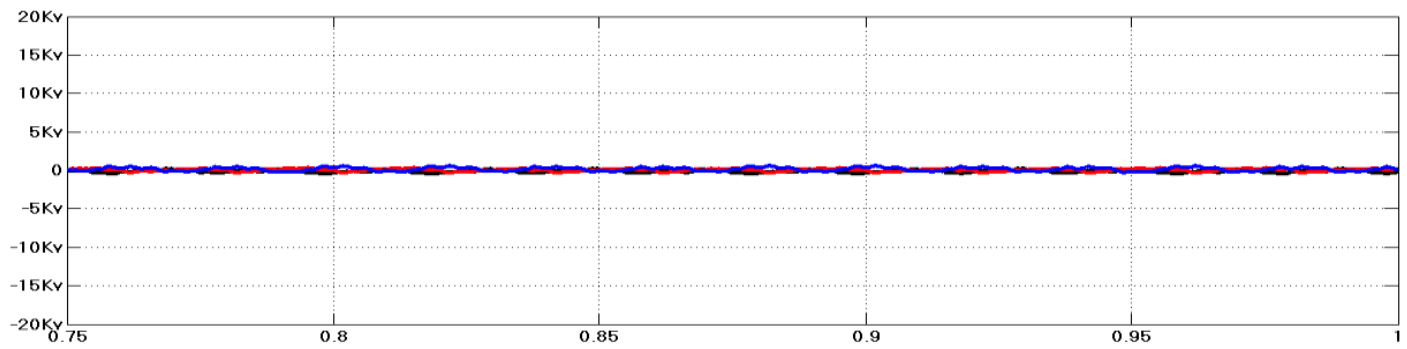

Fig.11. The compensation voltage by the DVR.

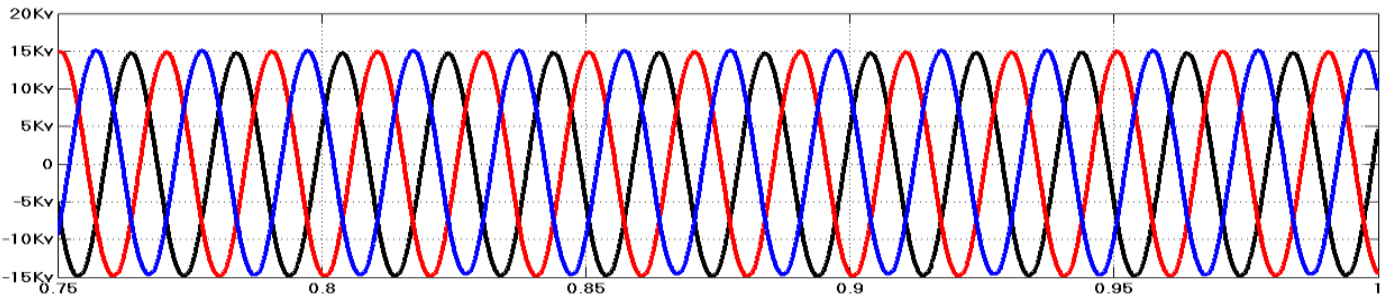

Fig.12. The load voltage at the third feeder (with DVR).

ii)Double line to ground fault

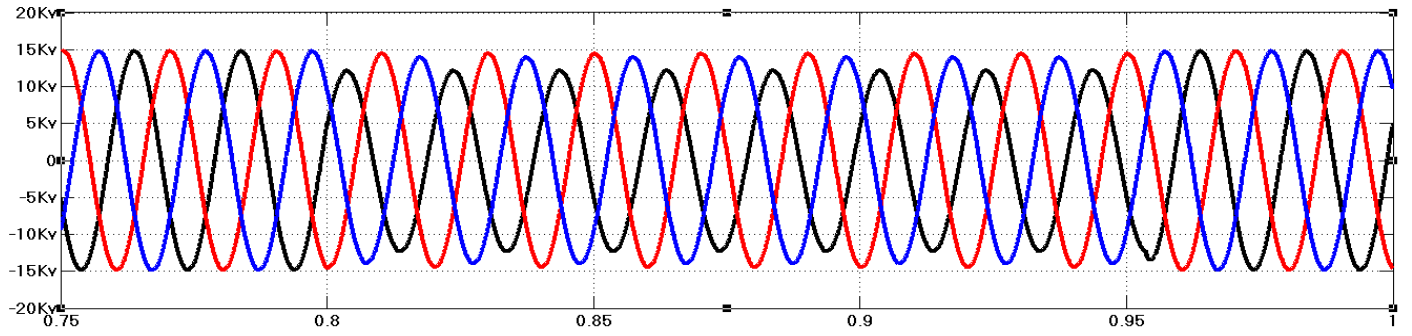

Fig.13. The load voltage at the second feeder (without DVR).

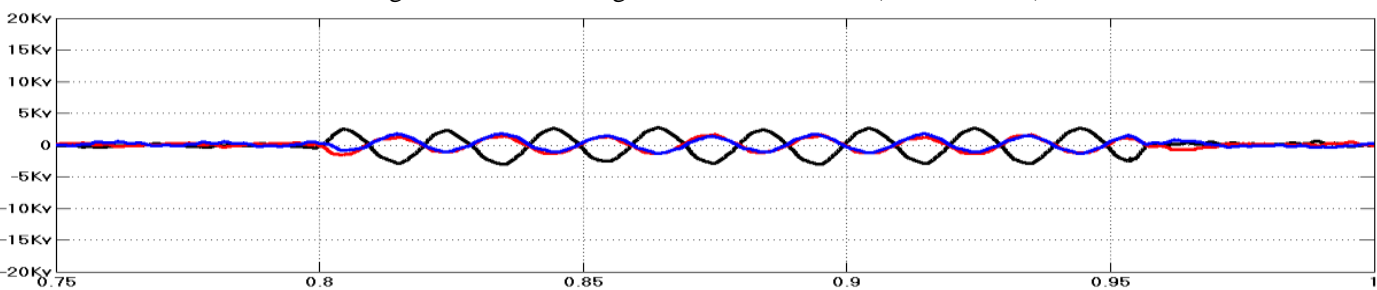

Fig.14. The compensation voltage by the DVR.

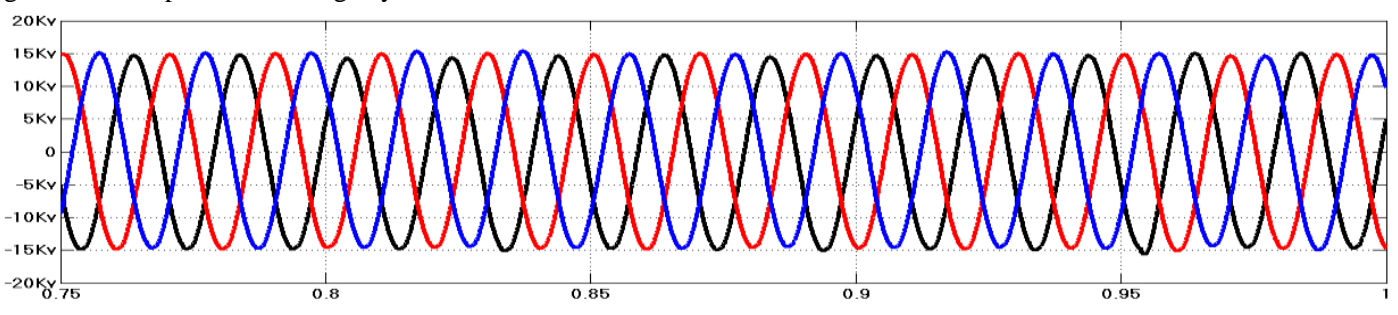

Fig.15. The load voltage at the third feeder (with DVR).

iii)Three phase to ground fault 


\section{WJES Wasit tournal

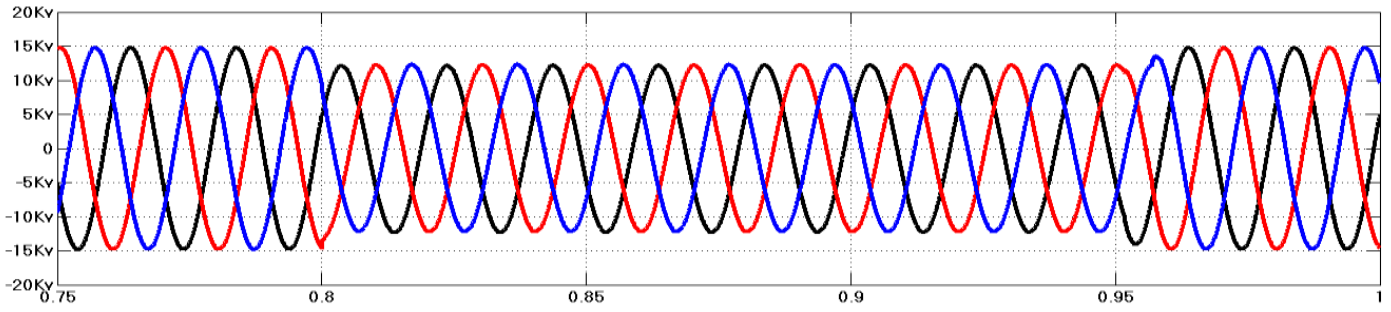

Fig.16. The load voltage at the second feeder (without DVR).

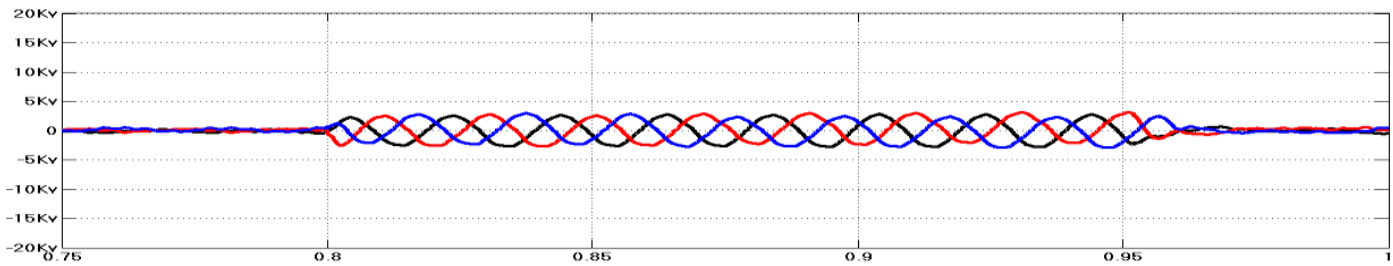

Fig. 17. The compensation voltage by the DVR.

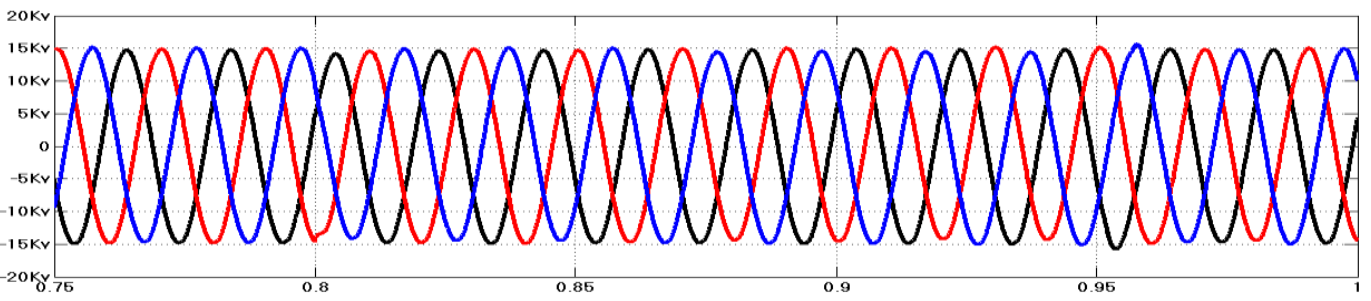

Fig.18. The load voltage at the third feeder (with DVR).

Different power disturbances like 50\%sag, $150 \%$ swell and voltage interruption have been simulated for a period of $0.15 \mathrm{~s}$ from $0.8 \mathrm{~s}$ to $0.95 \mathrm{~s}$ to see the function of the DVR based on Fuzzy Neural controller in protecting the load from voltage disturbances as depicted in figures below:

iv) $50 \% \mathrm{Sag}$

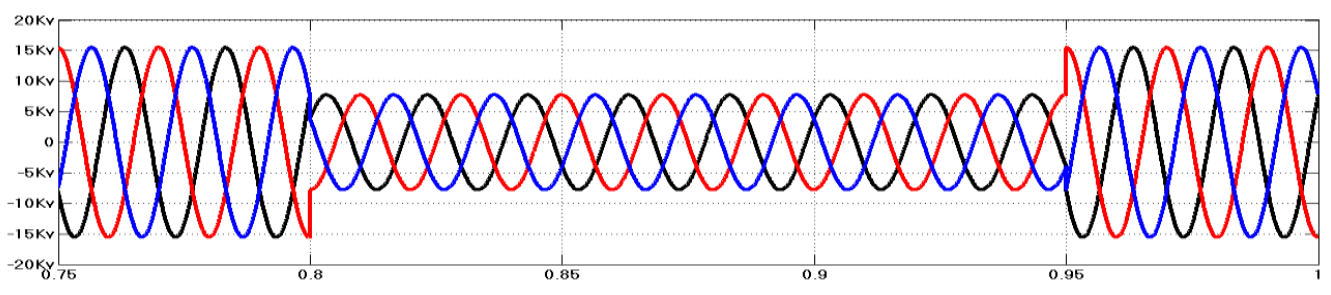

Fig.19: The load voltage at the second feeder (without DVR). 


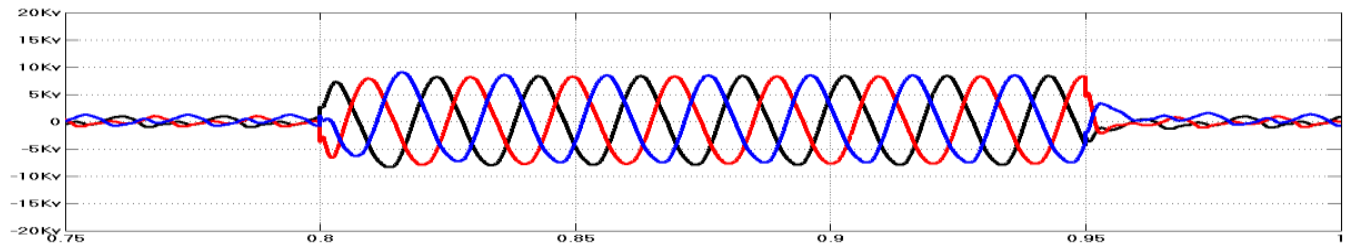

Fig.20. The compensation voltage by the DVR.

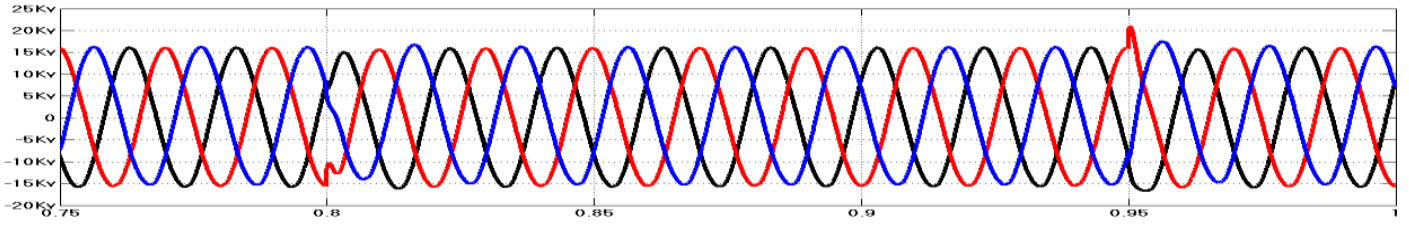

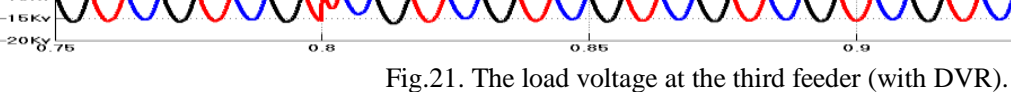

vi) $150 \%$ Swell
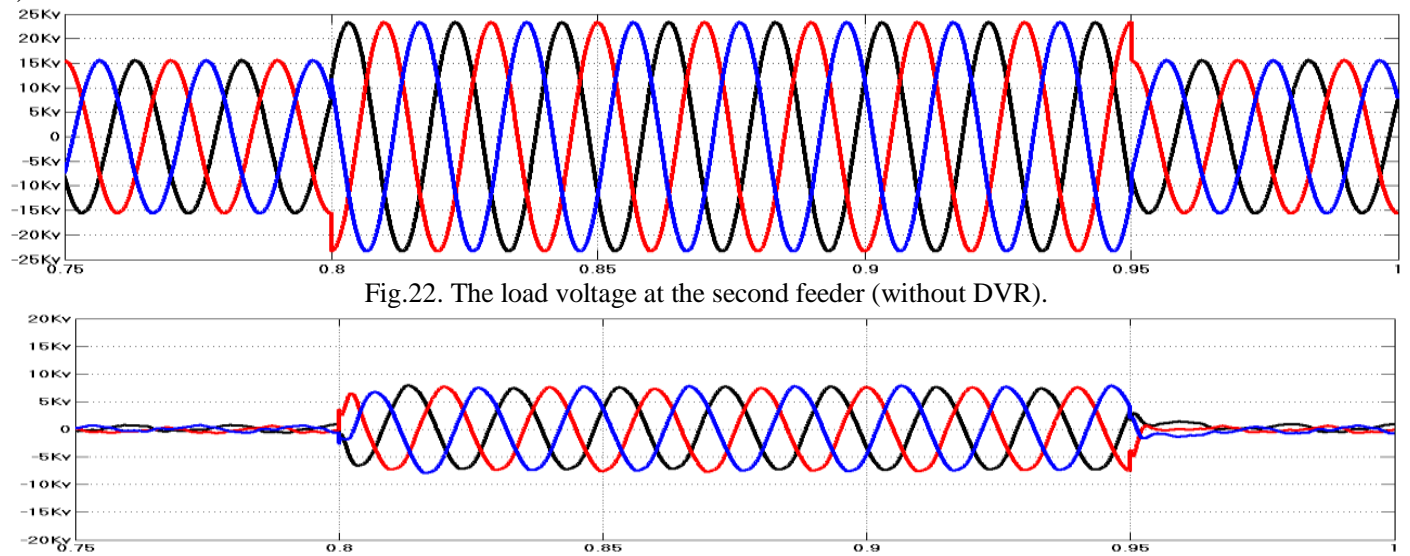

Fig.23. The compensation voltage by the DVR.

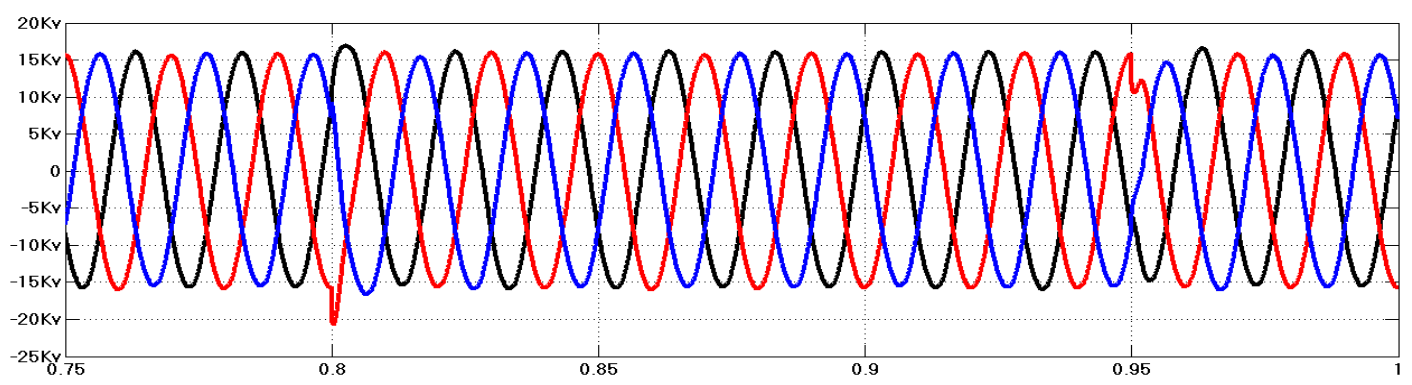

Fig.24. The load voltage at the third feeder (with DVR).

iiv) Voltage interruption

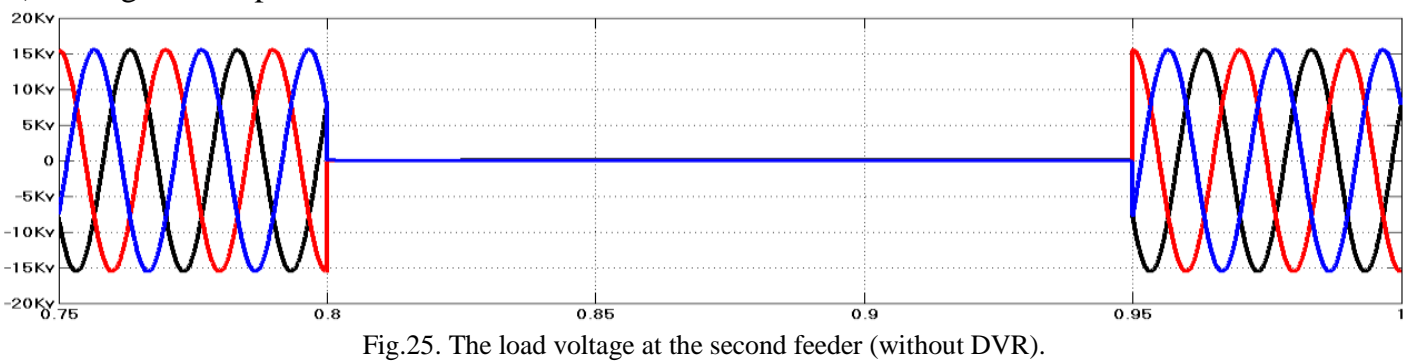




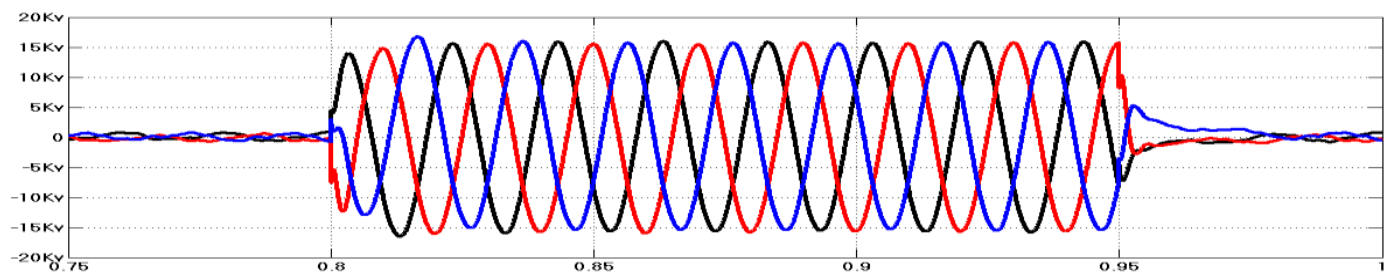

Fig.26. The compensation voltage by the DVR.

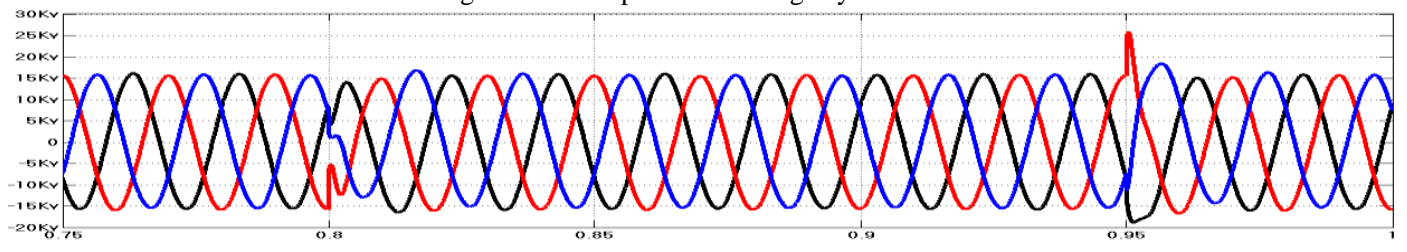

Fig.27. The load voltage at the third feeder (with DVR).

\section{CONCLUSIONS}

This paper deals with mitigating the power quality problems such as sag, swell, and voltage interruption. The custom power devices are used to enhance power transfer capabilities and stability margins of a transmission line. Dynamic Voltage Restorer is one of the most effective and efficient custom power devices due to the smaller size, lower cost, and the dynamic response. DVR can solve the voltage disturbances to protect the sensitive load in distribution system. The control strategy of the DVR plays an important role in its performance, that is any incorrect injection or delay in the process would be dangerous to the sensitive loads. DVR based on conconventional controller (PI) is not suited for dealing with a non-linear system or system with ill-defined and uncertainty such as the DVR which is a non-linear in nature therefore it is preferred to control the DVR using Artificial intelligent techniques available. In a power system under fault conditions, required results are not possible, in many literatures the controller was based on a conventional control theory which requires necessary information under abnormal and faults conditions. To overcome these problems, therefore this work proposes an adaptive controller like Fuzzy Neural optimized fuzzy logic controller where the second and the fourth layers in this controller are adaptable with different power disturbances and faults conditions. The proposed DVR handles different power disturbances by injecting the appropriate voltage components to correct any fault situation that occurred in the load side to keep the load voltage constant and balanced at the nominal value. Artificial intelligent especially the adaptive methods such as Fuzzy Neural optimized Fuzzy Logic controller is a better way to solve a non-linear system control problems since they have learning capabilities and parallel processing. therefore it has received a large attention in the field of controller design. As shown from simulation results Fuzzy Neural controller succeeds in restoring the correct value of the load voltage under different fault conditions and voltage variations quickly and efficiently without any difficulties.

\section{REFERENCES}

[1] Pandu Sathish Babu Nagappan Kamaraj, C 2017 'Power Quality Enhancement Using Dynamic Voltage Restorer', Lambert academic publishing, chapter.1, pp.2

[2] Pandu Sathish Babu Nagappan Kamaraj, C 2017 'Power Quality Enhancement Using Dynamic Voltage Restorer', Lambert academic publishing, chapter.1, pp.12

[3] Khalghani, MR, Shamsi-Nejad, MA, Farshad, M \& Khooban, MH 2014, 'Modifying power quality's indices of load by presenting an adaptive method based on hebb learning algorithm for controlling DVR',Automatika, pp.153-161

[4] Ferdi, B, Benachaiba, C, Dib, S \& Dehini, R 2010, 'Adaptive PI control of dynamic voltage restorer using fuzzy logic', Journal of Electrical Engineering: Theory and Application vol. 1, no. 3, pp. 165-173. 
[5] Choi, S, Li, J \& Vilathgamuwa, M 2005, 'A generalized voltage compensation strategy for mitigating the impacts of voltage sags/swells', IEEE Power Engineering Society Winter Meeting, vol. 4, pp. 2967-2972.

[6] K. Ramakrishna \& G. Koti , HO 2011, 'Performance investigation of neural network based unified power-quality conditioner', IEEE Transaction on power delivery, vol. 26, no. 1, pp. 431-437.

[7] Kinhal, VG, Agarwal, P \& Gupta, HO 2011, 'Performance investigation of neural network based unified power-quality conditioner'. IEEE Transactions on power delivery, vol. 26, no. 1, pp. 431-437.

[8] Dip, S, Ferdi, B \& Benachaiba, C 2011, 'Adaptive Neuro Fuzzy Inference System based DVR Controller Design', Leonardo Electronic Journal of Partices and Technologieas, no. 18, pp.49-64.

[9] Aali, S \& Nazarpour, D 2011, 'Voltage quality improvement with neural network-based interline dynamic voltage restorer', Journal of Electrical Engineering \& Technology, vol. 6,no.6,pp.769-775

[10] Singh, M \& Chandra, A 2013, 'Real time implementation of ANFIS control for renewable interfacing inverter in 3P4W distribution network', IEEE Transactions on Industrial Electronics, vol. 60, no. 1, pp. 121-128.

[11] Amutha, N \& Kumar, BK 2013, 'Improvement fault ride-through capability of wind generation system using DVR' , Electrical Power and Energy System, vol. 46, no. 1 , pp. 326-333 .

[12] Pandu Sathish Babu Nagappan Kamaraj, C 2017, 'Power Quality Enhancement Using Dynamic Voltage Restorer' LAP LAMBERT 